\title{
Halo concentrations and weak-lensing number counts in dark energy cosmologies
}

\author{
M. Bartelmann ${ }^{1}$, F. Perrotta ${ }^{2,3}$, and C. Baccigalupi ${ }^{2,4}$ \\ 1 Max-Planck-Institut für Astrophysik, PO Box 1317, 85741 Garching, Germany \\ ${ }^{2}$ Lawrence Berkeley National Laboratory, 1 Cyclotron Road, Berkeley, CA 94720, USA \\ 3 Osservatorio Astronomico di Padova, Vicolo dell'Osservatorio 5, 35122 Padova, Italy \\ ${ }^{4}$ SISSA/ISAS, via Beirut 4, 34014 Trieste, Italy
}

Received 1 July 2002 / Accepted 16 September 2002

\begin{abstract}
We study the effects of a dark energy component with equation of state $p=w \rho$ with constant $w \geq-1$ on the formation of Cold Dark Matter (CDM) haloes. We find two main effects: first, haloes form earlier as $w$ increases, and second, the amplitude of the dark-matter power spectrum gets reduced in order to remain compatible with the large scale Cosmic Microwave Background (CMB) anisotropies. These effects counteract. Using recipes derived from numerical simulations, we show that haloes are expected to be up to $\sim 50 \%$ more concentrated in CDM models with quintessence compared to $\Lambda$ CDM models, the maximum increase being reached for $w \sim-0.6$. For larger $w$, the amplitude of the power spectrum decreases rapidly and makes expected halo concentrations decrease. Halo detections through weak gravitational lensing are highly sensitive to halo concentrations. We show that weak-lensing halo counts with the aperture-mass technique increase by a factor of $\sim 2$ as $w$ is increased from -1 to -0.6 , offering a new method for constraining the nature of dark energy.
\end{abstract}

Key words. cosmology: theory - dense matter - gravitational lensing

\section{Introduction}

In recent years, cosmology has seen increasing observational evidence for an accelerating phase of the cosmic expansion, most notably through the observations of distant type Ia supernovae (Perlmutter et al. 1999; Riess et al. 1998). This astonishing evidence motivated renewed interest in the properties of the energy density ascribed to the "vacuum". A vacuum energy component should account for both the accelerating expansion and for the residual $\sim 70 \%$ of the energy density required for reconciling the geometrical flatness required by Cosmic Microwave Background (CMB) observations (De Bernardis et al. 2002; Lee et al. 2001; Halverson et al. 2002) with the evidence of a low-density universe with $\Omega_{0} \sim 0.3$ (Percival et al. 2001).

While one of the historical candidates for such an energy density is the cosmological constant, introduced as a simple geometrical term in Einstein's equations, it is well known that it leads to serious and unsolved theoretical problems. The exceedingly low value of the vacuum energy density today, compared to that allowed by the most plausible theories of the early stage of the Universe, motivated the introduction of a more general concept now widely known as "dark energy".

Preceding the evidence for cosmic acceleration, a generalisation of the cosmological constant by means of a scalar field,

Send offprint requests to: M. Bartelmann,

e-mail: msb@mpa-garching.mpg.de now known as "quintessence", was proposed (Wetterich 1988; Ratra \& Peebles 1988). In this class of models, the dark energy is supposed to reside mostly in the potential energy of the field, which interacts only gravitationally with ordinary matter. The evolution is described by the ordinary Klein-Gordon equation. If the potential is flat enough, or if the motion of the field along its trajectory is sufficiently slow, a cosmological constant-like behaviour can be mimicked by the scaling of the energy density of the quintessence field.

For general forms of the scalar field potential, there exist attractor trajectories for the evolution of the background expectation value of the field. These trajectories are known as "tracking" (Steinhardt et al. 1999) and "scaling" (Liddle \& Scherrer 1999) solutions. They have been shown to alleviate, at least at a classical level, the fine-tuning required in the early Universe, when the typical energy scales were presumably comparable to the Planck scale, to generate a vanishing relic vacuum energy as it is observed today, 120 orders of magnitude smaller. However, these scenarios are not able to solve the coincidence problem, i.e. why we are living in the epoch in which dark energy and matter have roughly the same energy density. Despite some attempts at addressing this issue (Tocchini-Valentini \& Amendola 2002; Chiba 2001; Armendariz-Picon et al. 2001; Dodelson et al. 2000), it remains one of the greatest puzzles of modern cosmology.

For constraining the nature of the dark energy, an important step would be accomplished if parameters could be 
constrained which capture its most essential features. In particular, if the dark energy is modelled as a quintessence field in the tracking regime, the simplest description of its properties will require the use of only two parameters, i.e. its present energy density and the ratio between pressure and energy density in its equation of state. Generally, this ratio depends on time, as implied by the evolution according to the equation of motion. However, it can be shown that, in most simple models of quintessence involving an inverse power-law potential, the effect of a time variation of the equation of state can be neglected at low redshifts, when the field has settled on its tracking trajectory. In this case, the equation of state is simply related to the power with which the potential depends on the field itself. This simplification allows constructing a scheme for describing the dark energy behaviour at redshifts where interesting cosmological effects arise, such as the effect on the magnitude-redshift relation of type Ia supernovae (Perlmutter 1999; Riess 1998) and the effect on gravitational lensing of distant galaxies and quasars (Futamase \& Yoshida 2001).

Even though the dark energy dynamics has a geometrical effect on acoustic features of the CMB anisotropy (Baccigalupi et al. 2002; Doran et al. 2002; Corasaniti \& Copeland 2002), it is now commonly accepted that the most interesting properties of a dark energy component have to be probed by looking at processes occurring at low redshifts when it starts dominating the cosmic expansion.

Thus, one of the most powerful probes of the quintessence field results from its effects on cosmic structure formation, most notably on the background cosmology and the evolution of individual collapsing overdensities. First, a dark energy component affects the matter density of the background in which haloes form. Second, the amplitude of matter perturbations is sensitive to the presence of a dynamical vacuum energy, through the normalisation of the matter power spectrum to the large, unprocessed, scales probed by the large-scale CMB anisotropies. Third, changes in the background matter density induced by a dark energy component can seriously affect characteristic properties of collapsing structures. As shown by Łokas \& Hoffmann (2002), a substantial quintessence component changes the characteristic density of a forming dark matter halo.

In this paper, we study how quintessence affects the concentration of dark-matter haloes, and resulting changes in their weak-lensing efficiency. Weak gravitational lensing provides a powerful tool for mapping the large-scale mass distribution (see Mellier 1999a and Bartelmann \& Schneider 2001 for reviews), and the potential impact of dark energy on the weak lensing convergence power spectrum has already been recognised (see Mellier 1999b; Huterer 2002). We show in this paper that an energy density component with negative pressure affects weak lensing by dark-matter haloes not only through changes in the global properties of the Universe, but also by modifying their internal density concentration. The main idea is that dark energy affects structure growth and thus the time of halo formation. Since halo concentrations reflect the density of the Universe at their formation epoch, this affects halo mass distributions, and weak lensing provides methods for quantifying respective changes.
The paper is organised as follows. In Sect. 2, we describe the main effects of the dark energy on the cosmological growth factor, volume elements, and the normalisation of the darkmatter power spectrum. In Sect. 3 we compute the impact on halo concentration. In Sect. 4 we predict resulting effects on the weak-lensing aperture mass statistics, and Sect. 5 contains our conclusions.

\section{Cosmological implications of quintessence models}

We model the dark energy as a spatially homogeneous component, labelled $Q$, with constant equation of state parameterised by the ratio between the pressure $p_{\mathrm{Q}}$ and the energy density $\rho_{\mathrm{Q}}$, $w=p_{\mathrm{Q}} / \rho_{\mathrm{Q}}$. We neglect a possible time variation of $w$, as well as any effects possibly due to spatial inhomogeneities of the dark energy. Indeed, at least in most models proposed so far, the relevant cosmological effects of the dark energy compared to a cosmological constant are mainly related to its effective equation of state at redshifts when it is relevant for cosmic expansion, say $z \leq 5$, as we already noted in the introduction. Neglecting inhomogeneities of the dark energy is justified in the present context since they are likely to show relativistic behaviour on sub-horizon cosmological scales; indeed, the effective mass of the vacuum component, of the order of the critical density today, is extremely light compared to any other known massive particle, so that quintessence clustering occurs only on scales larger than or equal to the horizon size (Ma et al. 1999). It can also be shown formally that a minimally coupled quintessence field has a relativistic effective sound speed $(\mathrm{Hu}$ 1998), so that its fluctuations are damped out on the scales in which we are interested here.

Assuming the equation of state $p_{\mathrm{Q}}=w \rho_{\mathrm{Q}}$, the adiabatic equation implies

$\rho_{\mathrm{Q}}=\rho_{\mathrm{Q}, 0} a^{-3(1+w)}$,

where $\rho_{\mathrm{Q}, 0}$ is the quintessence energy density today, and $a$ is the cosmic scale factor normalised to unity at the present epoch. Friedmann's equation can then be written,

$H^{2}(t)=H_{0}^{2}\left[\Omega_{\mathrm{Q}} a^{-3(1+w)}+\Omega_{0} a^{-3}+\left(1-\Omega_{\mathrm{Q}}-\Omega_{0}\right) a^{-2}\right]$,

where $\Omega_{\mathrm{Q}}$ is the quintessence density parameter at the present epoch, i.e. $\rho_{\mathrm{Q}, 0}$ divided by the critical energy density today. The density parameter for non-relativistic matter is $\Omega_{0}$ today. Obviously, for $w=-1$, Friedmann's equation for a cosmological constant is retained. Unless stated otherwise, we will assume in this paper that the curvature of spatial hypersurfaces is zero, thus $\Omega_{\mathrm{Q}}+\Omega_{0}=1$, and the curvature term in (2) vanishes.

The quintessence term in (2) has two immediate consequences relevant for our purposes. First, the way how density inhomogeneities grow is modified, and second, the cosmic volume per unit redshift changes.

In linear theory, the density contrast $\delta$ of matter perturbations grows according to

$\ddot{\delta}+2 \frac{\dot{a}}{a} \dot{\delta}-4 \pi G \rho \delta=0$, 


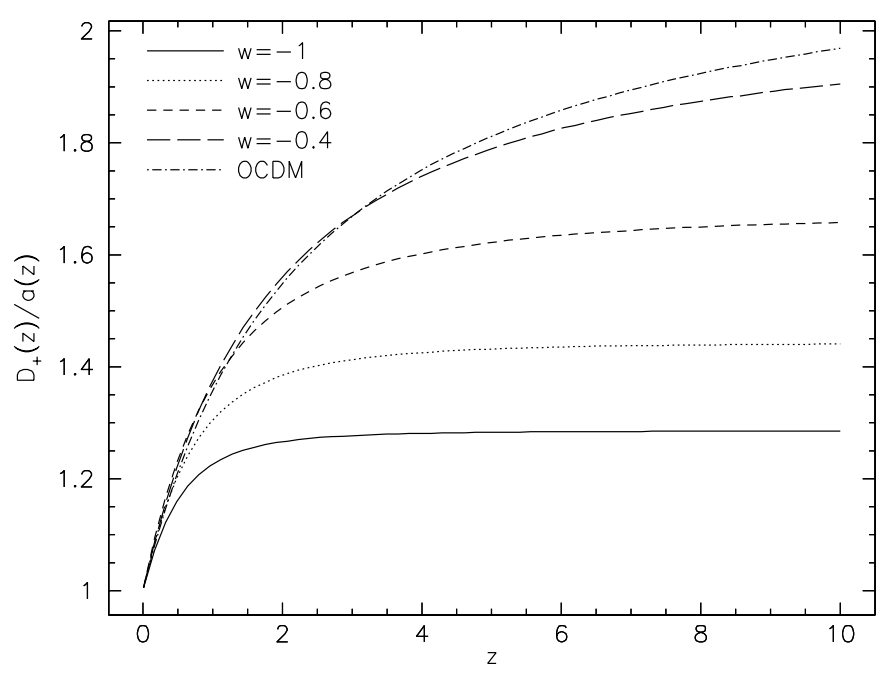

Fig. 1. Growth factor $D_{+}(z)$ as a function of redshift for five different cosmological models as indicated. The growth factor is normalised to unity at the present epoch, and divided by the scale factor to emphasise the differences between the models. With increasing $w$, the growth factor increases towards its value for the open model with $\Omega_{\mathrm{Q}}=0$.

where $\rho$ is the total matter density and the dots denote derivatives with respect to time. For dark-energy models with $w=-1$ or $w=-1 / 3$, the solution can be written in the form

$\delta \propto \frac{\dot{a}}{a} \int_{0}^{a} \frac{\mathrm{d} a}{\dot{a}^{3}}$

(Heath 1977; see also Chap. 15 of Peacock 1999). The linear growth of $\delta$ is generally described by the so-called growth factor $D_{+}(a)$, which is commonly normalised either to unity at $a=1$, or such that it rises proportional to $a$ for $a \ll 1$. We plot in Fig. 1 the growth factor as a function of redshift $z$, normalised to unity today and divided by $a$. The normalisation ensures that $D_{+} / a$ goes to unity for $z \rightarrow 0$. In an Einsteinde Sitter model, $D_{+}=a$, thus the curves show how much earlier structures grow in the assumed model universes compared to an Einstein-de Sitter model. Curves are shown for a variety of cosmological models. All of them have $\Omega_{0}=0.3$ and either $\Omega_{\mathrm{Q}}=1-\Omega_{0}$ or, for comparison, $\Omega_{\mathrm{Q}}=0$.

The solid curve in Fig. 1 shows the growth factor for a model universe with a cosmological constant $(w=-1)$. Going back in time, $D_{+} / a$ rises from unity to $\sim 1.3$ and turns flat near $z \sim \Omega_{0}^{-1} \sim 3$. This means that structures start forming earlier in this model compared to an Einstein-de Sitter model, but the growth slows down considerably at redshifts smaller than $z \sim 3$. For the low-density open model without quintessence (labelled $\Omega_{\mathrm{Q}}=0$ ), $D_{+} / a$ keeps rising as $z$ increases. At redshift 5, for instance, the amplitude of structures is 1.4 times higher than in the cosmological-constant model. Increasing $w$ interpolates between the open and the flat model with cosmological constant. Thus, keeping $\Omega_{0}$ and $\Omega_{\mathrm{Q}}$ fixed, structures form earlier for larger values of $w$, approaching the growth behaviour for low-density open models without quintessence or cosmological constant.

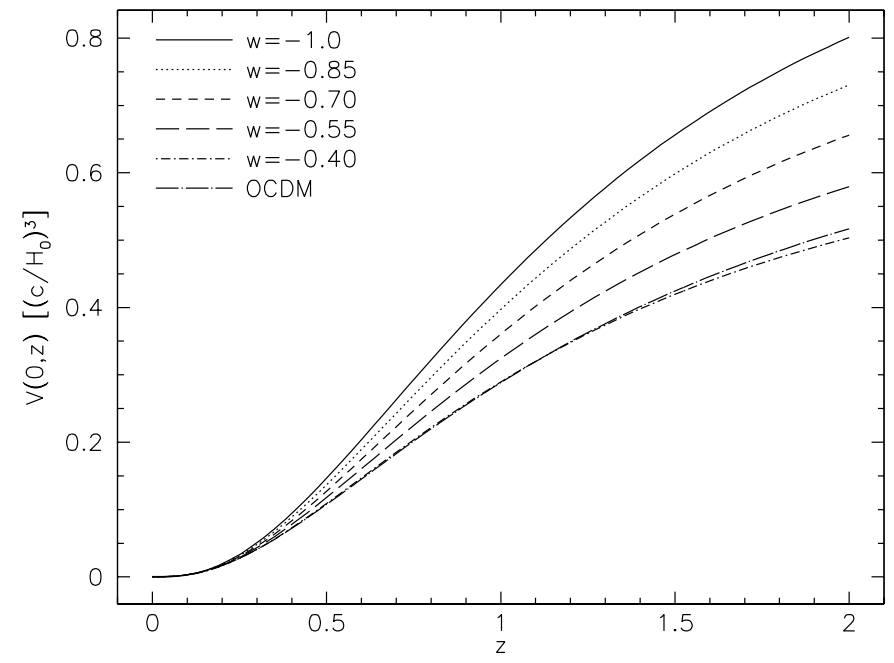

Fig. 2. Cosmic volume in units of the Hubble volume between redshift zero and $z$ for six different cosmological models as indicated. With increasing $w$, the cosmic volume decreases towards its value for the open model with $\Omega_{\mathrm{Q}}=0$.

A similar interpolation is seen in the behaviour of the cosmic volume per unit redshift. Figure 2 shows

$V(z)=\int_{0}^{z} \mathrm{~d} z^{\prime} 4 \pi D^{2}\left(z^{\prime}\right)\left|\frac{c \mathrm{~d} t}{\mathrm{~d} z}\right|\left(z^{\prime}\right)$,

where $D(z)$ is the angular-diameter distance between redshifts 0 and $z$, thus $V(z)$ is the proper volume of a sphere of "radius" $z$ around the observer. Similar to the behaviour of the growth factor, dark-energy models interpolate between the two limiting curves, where the model with cosmological constant has the largest and the model without cosmological constant has the smallest volume.

Here and below, we keep the cosmological parameters fixed at $\Omega_{0}=0.3, \Omega_{\mathrm{Q}}=0.7$. The Hubble constant is $H_{0}=$ $100 h \mathrm{~km} \mathrm{~s}^{-1} \mathrm{Mpc}^{-1}$ with $h=0.7$.

In order to describe the formation of dark-matter haloes, we need to specify the power spectrum of dark-matter fluctuations. We choose the cold dark matter (CDM) model, whose transfer function was given by Bardeen et al. (1986), and we set the index of the primordial power spectrum to the HarrisonZel'dovich value of $n=1$. Then, the power spectrum has two free parameters, the shape parameter $\Gamma$ which locates its maximum, and the amplitude. For the shape parameter, we assume $\Gamma=\Omega_{0} h$ as suggested by theory and in agreement with observations of the galaxy power spectrum. For our model for dark energy, the transfer function by Bardeen et al. is applicable because dark energy does not cluster on the relevant scales, and the shape parameter $\Gamma$ is set by the scale of matter-radiation equality, which is unaffected by $w$ (cf. Wang \& Steinhardt 1998).

The amplitude of the power spectrum needs to be chosen so that certain observations can be reproduced. It is an important constraint that the abundance of rich galaxy clusters in our cosmic neighbourhood be reproduced. Since the galaxy-cluster mass function falls very steeply at the high-mass end, small changes in the amplitude of the power spectrum lead to large 


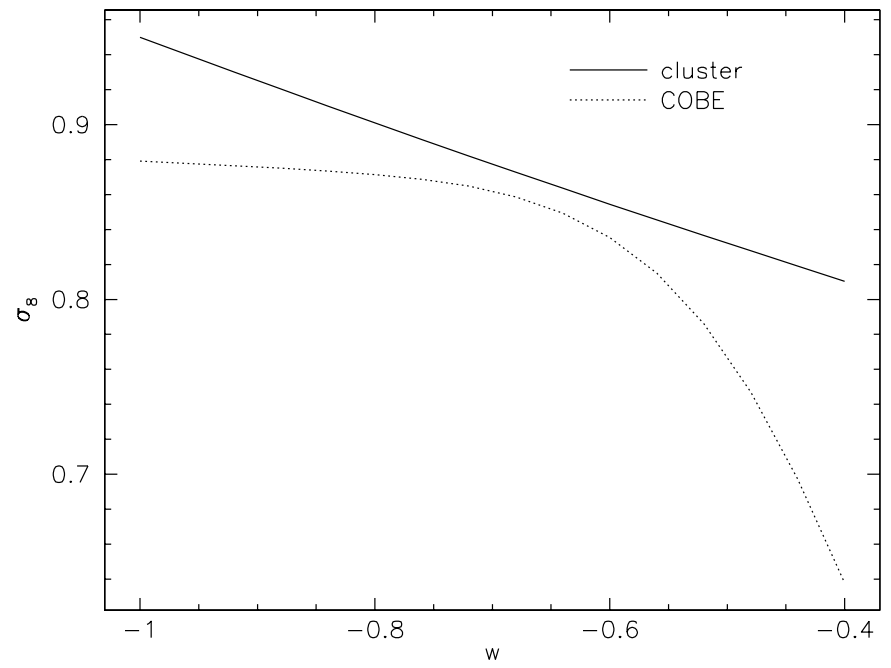

Fig. 3. The normalisation of the power spectrum, expressed in terms of $\sigma_{8}$, is shown as a function of $w$ for two different normalisation methods. The solid line shows the cluster-abundance normalisation derived by Wang \& Steinhardt, the dotted line shows $\sigma_{8}$ as constrained by the COBE-DMR data. While $\sigma_{8}$ is almost constant in the latter case for $w \lesssim-0.6$, it drops rapidly for larger $w$ because of the increasingly strong integrated Sachs-Wolfe effect. The uncertainty of $\sigma_{8}$ determined from COBE data is approximately $7 \%$ (Bunn \& White 1997).

changes in the cluster number density, thus the amplitude is in principle well constrained by the cluster abundance. However, for that normalisation procedure, rich galaxy clusters are identified by their X-ray emission, thus the reliability of the normalisation depends on how well models can describe the X-ray properties of the intracluster gas.

We saw before that structure starts forming earlier in quintessence models with $w>-1$ compared to models with cosmological constant. Galaxy clusters forming earlier are hotter because of the higher mean density of their surroundings. Reproducing the current number density of X-ray selected clusters thus requires a power-spectrum amplitude which decreases with increasing $w$ (Wang \& Steinhardt 1998). Figure 3 shows their results, expressing the power-spectrum amplitude in terms of the rms fluctuation amplitude $\sigma_{8}$ on a physical scale of $8 h^{-1} \mathrm{Mpc}$.

The power spectrum of density perturbations can be normalised by the $\mathrm{CMB}$ anisotropies on large angular scales, corresponding to physical scales which were larger than the horizon at decoupling. The only available data on those scales are those of the Differential Microwave Radiometer (DMR) experiment on board the COsmic Background Explorer satellite (COBE, Bennett et al. 1996), but much improved data are being taken by the Microwave Anisotropy Probe (MAP, http://map.gsfc.nasa.gov/), and will be taken by the Planck satellite (http://astro. estec. esa.nl/SA-general / Projects / Planck/). Subdegree CMB anisotropies have been measured by several experiments (De Bernardis et al. 2001; Lee et al. 2001; Halverson et al. 2002). However, on these angular scales, the main effect of increasing $w$ above -1 is to rigidly shift acoustic peaks toward larger angular scales (see Baccigalupi et al. 2002 and references therein), having almost no effect on the overall normalisation of the spectrum.

Normalising the power spectrum according to the COBE measurements fixes the amplitude of the power spectrum on its large-scale end. As $w$ increases at fixed $\Omega_{0}$ and $\Omega_{\mathrm{Q}}$, dark energy dominates cosmic expansion earlier compared to a model with cosmological constant having the same energy density today. This has the effect of enhancing the dynamics of the gravitational potential due to the change in the cosmic equation of state, thus increasing the Integrated Sachs-Wolfe (ISW) effect on COBE scales. While this trend is gentle for $w \lesssim-0.6$, it steepens for larger $w$, as shown by the dotted line in Fig. 3 .

This non-linear behaviour of $\sigma_{8}$ reflects the sensitivity of the ISW effect (and thus the normalisation) to the redshift $z_{\mathrm{Q}}$ at which dark energy starts dominating over matter. It is easy to show that this is determined by the power law $1+z_{\mathrm{Q}}=$ $\left(\Omega_{0} / \Omega_{\mathrm{Q}}\right)^{-1 / 3 w}$. We recall that, although we consider values of $w$ up to -0.4 for the sake of generality, values much higher than -0.6 are not interesting in the framework of dark-energy models. Indeed, $w$ larger than -0.48 is insufficient to provide cosmic acceleration if $\Omega_{Q}=0.7$ as we assume in this work. In addition, the sharp decrease in Fig. 3 due to the ISW leads to a decrease of the acoustic peaks in the CMB power spectrum below the level observed by experiments operating on sub-degree angular scales.

In order to avoid the uncertainties in $\sigma_{8}$ due to the uncertainties in modelling the X-ray cluster population, we choose the COBE normalisation for our study. We adopt the normalisation method by Bunn \& White (1997), which has a 7\% accuracy. This procedure exploits a maximum likelihood approach for reproducing the measured CMB anisotropy power once the sky regions affected by the Galactic signal have been cut out. The CMB anisotropy can be expressed as a line-ofsight integral of the perturbation power spectrum weighted with suitable geometric functions, implemented in the CMBFAST code (Seljak \& Zaldarriaga 1996). For taking the dark energy component into account, we use a modified version of CMBFAST (see Baccigalupi et al. 2000). Since halo properties are determined by the small-scale end of power spectrum while the COBE normalisation fixes its large-scale end, quantitative results will sensitively depend on the index $n$ of the power spectrum.

An entirely different and perhaps more direct way of normalising the power spectrum has become feasible recently. Large-scale density fluctuations differentially deflect light on its way from distant sources to us. The gravitational tidal field of the matter inhomogeneities coherently distorts the images of faint background galaxies. Albeit weak, this cosmic shear effect has recently been measured successfully by several groups, whose results agree impressively although different telescopes, observational parameters and data-reduction techniques were used (van Waerbeke et al. 2000; Bacon et al. 2000; Kaiser et al. 2000; Wittman et al. 2000; Maoli et al. 2001; van Waerbeke et al. 2001). Since gravitational lensing depends only on the matter distribution and not on its composition or physical state, cosmic shear should provide one of the cleanest ways for constraining the power-spectrum amplitude. 


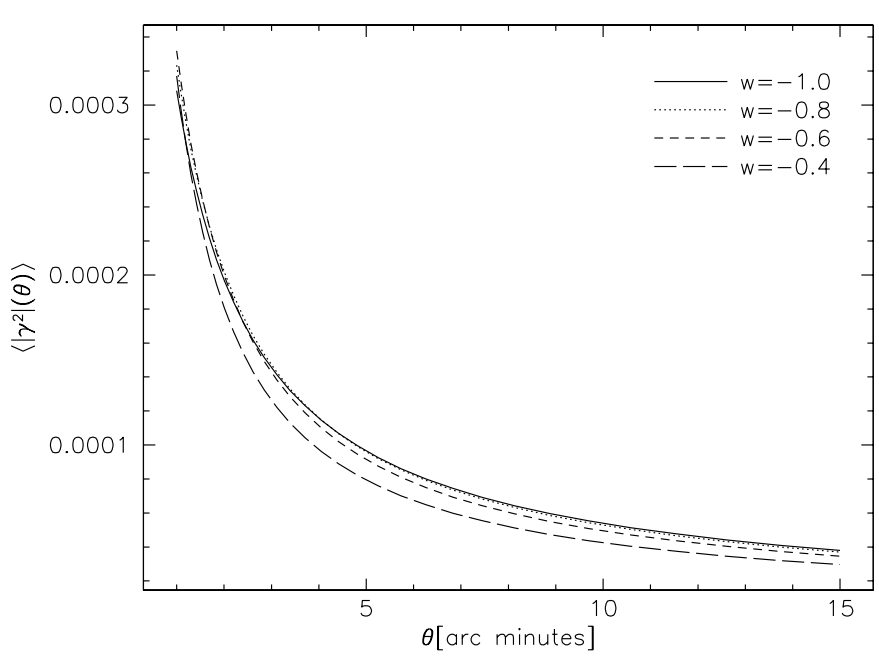

Fig. 4. The squared cosmic shear $\left\langle\left|\gamma^{2}\right|\right\rangle(\theta)$, averaged in apertures of radius $\theta$, is shown as a function of $\theta$ for five different cosmological models as indicated. A source redshift distribution with mean redshift $\sim 0.9$ was assumed for the plot. Changing $w$ has very little effect on the curves, implying that the constraints on $\sigma_{8}$ derived from cosmic-shear measurements are insensitive to $w$.

Cosmic-shear measurements have been shown to agree very well with the expectations in a $\Lambda$ CDM universe. Fixing the shape parameter to $\Gamma=0.21$, the measurements require $\Omega_{0}<0.4$ and $\sigma_{8}>0.7$ at $95 \%$ confidence (van Waerbeke et al. 2001). Note, however, that these constraints depend on the nonlinear evolution of the power spectrum which has some uncertainties.

This result is almost completely insensitive to $w$ in quintessence models. In Fig. 4, we show the rms cosmic shear in apertures of radius $\theta$ as a function of $\theta$ for four models differing by $w$, as indicated. The power spectrum was normalised to reproduce the COBE measurements, and its nonlinear evolution was approximated using the fitting formulae by Peacock \& Dodds (1996). Following van Waerbeke et al. (2001), we adopted the source redshift distribution shown in (9) below with parameters $z_{0}=0.8$ and $\beta=1.5$. The curves are fully compatible with the current cosmic-shear measurements. Moreover, they are much closer to each other than the typical uncertainty of the measurements, which is mainly due to the fact that cosmic shear is most sensitive to structures below redshift $\sim 0.5$ where the differences between the models are small. This allows two conclusions. First, the COBE normalisation is not in conflict with the measured cosmic shear for all interesting values of $w$, otherwise the curves would disagree with the measurements; and second, the constraint on $\sigma_{8}$ derived from current cosmic-shear data is independent of $w$ because the curves do not significantly change with $w$.

\section{Halo concentrations in dark energy models}

Numerical simulations of cosmic structure formation show consistently that the density profiles of dark-matter haloes can be described by a two-parameter family of models. Far outside a scale radius $r_{\mathrm{s}}$, the profiles fall off proportional to $r^{-3}$, while they are cuspy but considerably flatter well within $r_{\mathrm{s}}$. The exact inner profile slope is under debate. The second free parameter besides the scale radius is a characteristic density scale $\rho_{\mathrm{s}}$. We adopt the density profile suggested by Navarro et al. (1995),

$$
\rho(r)=\frac{\rho_{\mathrm{s}}}{\left(r / r_{\mathrm{s}}\right)\left(1+r / r_{\mathrm{s}}\right)^{2}} .
$$

The two parameters $r_{\mathrm{s}}$ and $\rho_{\mathrm{s}}$ are not independent. Haloes are commonly parameterised by their virial mass $M$. For a given cosmology, this also defines their virial radius $R$. Numerical simulations show that the scale radius depends on the mass such that the halo concentration $c=R / r_{\mathrm{s}}$ is a characteristic function of mass. Given $M, R$ and $c$, the density scale $\rho_{\mathrm{s}}$ is fixed. For definiteness, we parameterise halo masses consistently by $M_{200}$, i.e. masses enclosed in spheres with radius $R_{200}$ in which the average density is 200 times the critical density.

Numerically simulated haloes turn out to be the more concentrated the less massive they are. Since less massive haloes form earlier than more massive ones in hierarchical models of structure formation, this is interpreted assuming that the central density of a halo reflects the mean cosmic density at the time when the halo formed (e.g. Navarro et al. 1997).

Several algorithms based on this assumption have been suggested for describing the concentration of dark-matter haloes. Originally, Navarro et al. (1997) devised the following approach. A halo of mass $M$ is first assigned a collapse redshift $z_{\text {coll }}$ defined as the redshift at which half of the final halo mass is contained in progenitors more massive than a fraction $f_{\mathrm{NFW}}$ of the final mass. Then, the density scale of the halo is assumed to be some factor $C$ times the mean cosmic density at the collapse redshift. They recommended setting $f_{\mathrm{NFW}}=0.01$ and $C=3 \times 10^{3}$ because their numerically determined halo concentrations were well fit assuming these values.

Bullock et al. (2001) noticed that halo concentrations change more rapidly with halo redshift than the approach by Navarro et al. (1997) predicts. They suggested a somewhat simpler algorithm. Haloes are assigned a collapse redshift defined such that the non-linear mass scale at that redshift is a fraction $f_{\mathrm{B}}$ of the final halo mass. The halo concentration is then assumed to be a factor $K$ times the ratio of the scale factors at the redshift when the halo is identified and at the collapse redshift. Comparing with numerical simulations, they found $f_{\mathrm{B}}=0.01$ and $K=4$.

Yet another algorithm was suggested by Eke et al. (2001). They assigned the collapse redshift to a halo of mass $M$ by requiring that the suitably defined amplitude of the linearly evolving power spectrum at the mass scale $M$ equals a constant $C_{\mathrm{ENS}}^{-1}$. Numerical results are well represented setting $C_{\mathrm{ENS}}=28$.

Since we consistently use $R_{200}$ and $M_{200}$ for parameterising haloes, we need to convert masses and concentration parameters from the slightly different definitions introduced by Bullock et al. and Eke et al. In particular, this requires us to iteratively compute the concentration parameter according to our definition. Moreover, the algorithms implicitly use the mean overdensity of virialised haloes, $\Delta_{\mathrm{V}}$, and the linear overdensity of collapsed haloes, $\delta_{\mathrm{c}}$. These parameters depend on cosmology and on the dark energy parameter $w$. We compute them using the formulae given in Łokas \& Hoffmann (2002). 


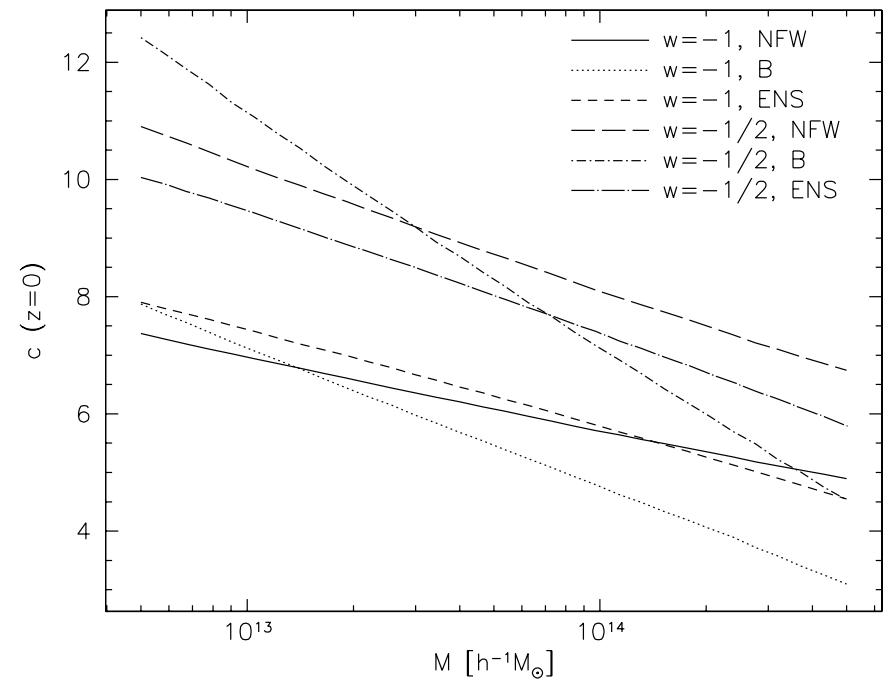

Fig. 5. Halo concentration parameters $c$ as functions of halo mass $M_{200}$. Results obtained from three different prescriptions for calculating the concentration are shown; these are the prescriptions from Navarro et al. (NFW), Bullock et al. (B) and Eke, Navarro \& Steinmetz (ENS). Curves are shown for the $\Lambda$ CDM model $(w=-1)$ and for a quintessence model with $w=-1 / 2$. Irrespective of the prescription, the concentration parameters are higher in the quintessence model than in the $\Lambda \mathrm{CDM}$ model for all halo masses.

Although halo concentrations produced by these different algorithms differ in detail, they have in common that haloes forming earlier are more concentrated. Taken together with the earlier result that haloes form earlier in dark energy models with $w>-1$ than in $\Lambda$ CDM models, this implies that haloes are expected to be more concentrated in models with $w>-1$. Figure 5 illustrates this. Halo concentrations computed with the three different algorithms are plotted as functions of halo mass for redshift zero. Since less massive haloes start forming earlier than more massive ones in hierarchical models like CDM, the concentration decreases with halo mass. Curves are shown for models with $w=-1$ and $w=-1 / 2$, keeping all other parameters fixed. At $M \sim 5 \times 10^{13} h^{-1} M_{\odot}$, for instance, halo concentrations are approximately $50 \%$ higher in the dark energy compared to the $\Lambda \mathrm{CDM}$ model.

Concentrations of haloes with fixed mass $M$ identified at $z>0$ tend to be smaller than at redshift zero. This does not contradict the finding that haloes forming earlier are more concentrated because haloes of a given mass $M$ at $z>0$ have larger mass at redshift zero, thus the change of halo concentrations with redshift for fixed mass reflects their change with mass for fixed redshift. Likewise, the trend of increasing concentrations with increasing $w$ remains also at higher redshifts.

Figure 6 shows halo concentrations as functions of mass derived from the algorithm suggested by Bullock et al. for five different choices of $w$, keeping all other parameters fixed. As $w$ increases away from -1 , concentrations first increase for all halo masses shown. A maximum is reached for $w \sim-0.6$. As $w$ is raised further, concentrations decrease rapidly. This is an effect of the power-spectrum normalisation. As $w$ increases, $\sigma_{8}$ must decrease because otherwise the normalisation constraints would be violated; either there would be too many hot galaxy

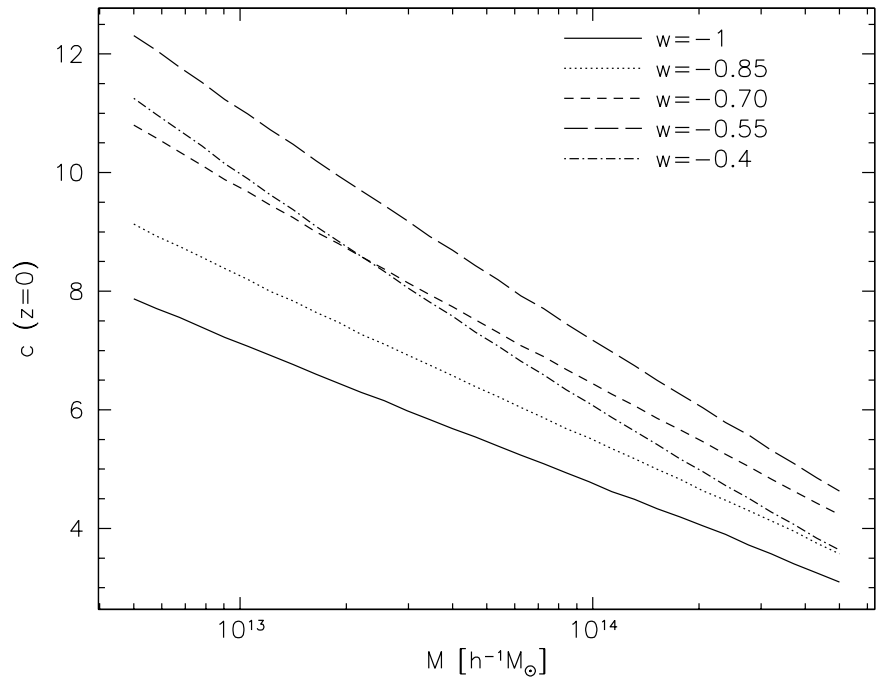

Fig. 6. Halo concentrations obtained from the algorithm described by Bullock et al. as functions of halo mass $M_{200}$ for five different cosmological models as indicated. With increasing $w$, the concentration increases until $w \gtrsim-0.6$, and drops rapidly as $w$ increases further.

clusters, or the secondary CMB anisotropies caused by the integrated Sachs-Wolfe effect would exceed the COBE measurements. As $\sigma_{8}$ is lowered, haloes form later, thus counter-acting the earlier increase of the growth factor in quintessence models. Adopting the COBE normalisation, the maximum effect is achieved just before the integrated Sachs-Wolfe effect sets in strongly at $w \gtrsim-0.6$, cf. Fig. 3 .

\section{Weak lensing and halo counts}

The impact of dark energy on halo concentrations should cause an impact on many observable quantities. As an illustration, we will now describe how the number of haloes detectable through their weak gravitational lensing effect change with $w$.

The gravitational tidal field of individual sufficiently massive haloes imprints coherent distortions on the images of faint background galaxies in their neighbourhood. Haloes can thus be detected searching for their characteristic signature on the appearance of the background galaxy population. A sensitive and convenient technique for halo detection, the aperture mass technique, has been suggested by Schneider (1996; see also Kruse \& Schneider 1999).

Consider a circular aperture of angular radius $\theta$. The aperture mass is defined as a weighted integral of the lensing convergence $\kappa$ across the aperture,

$M_{\mathrm{ap}}(\theta)=\int \mathrm{d}^{2} \vartheta \kappa(\boldsymbol{\vartheta}) U(|\boldsymbol{\vartheta}|)$

The convergence is the surface mass density scaled by its critical value for strong lensing,

$\kappa=\frac{\Sigma}{\Sigma_{\mathrm{cr}}}, \quad \Sigma_{\mathrm{cr}}=\frac{c^{2}}{4 \pi G} \frac{D_{\mathrm{s}}}{D_{\mathrm{d}} D_{\mathrm{ds}}}$,

where $D_{\mathrm{d}, \mathrm{ds}, \mathrm{s}}$ are the angular-diameter distance from the observer to the lens, the lens to the source, and the observer to the source, respectively. 
Through $\Sigma_{\text {cr }}$, the aperture mass (7) depends on the source redshift. We compute mean aperture masses by averaging $M_{\text {ap }}$ over the normalised source-redshift distribution

$p\left(z_{\mathrm{s}}\right)=\frac{\beta}{z_{0}^{3} \Gamma(3 / \beta)} \exp \left[-\left(\frac{z}{z_{0}}\right)^{\beta}\right]$

with $z_{0}=1$ and $\beta=1.5$, implying a mean source redshift of $\sim 1.5$ (cf. Smail et al. 1995; Cohen et al. 2000).

If the weight function $U(|\boldsymbol{\vartheta}|)$ is compensated,

$\int \mathrm{d}^{2} \vartheta U(|\boldsymbol{\vartheta}|)=0$,

the aperture mass $M_{\text {ap }}$ can be written as a (differently) weighted integral across the aperture of the tangential component of the shear with respect to the aperture centre. Thus, $M_{\text {ap }}$ is a directly observable quantity.

Halo detection can then proceed as follows. An aperture of given radius is shifted across a wide and sufficiently deep field. At all aperture positions, the aperture mass is determined. Potential haloes are located where $M_{\text {ap }}$ exceeds a certain threshold.

The signal-to-noise ratio of an aperture-mass measurement is given by $\mathcal{S}=M_{\mathrm{ap}} \sigma_{\mathrm{M}}^{-1}$ with the dispersion

$\sigma_{\mathrm{M}}(\theta)=0.016\left(\frac{n_{\mathrm{g}}}{30 \operatorname{arcmin}^{2}}\right)^{-1 / 2}\left(\frac{\sigma_{\epsilon}}{0.2}\right)\left(\frac{\theta}{1^{\prime}}\right)^{-1}$,

where $n_{\mathrm{g}}$ is the number density of faint background galaxies and $\sigma_{\epsilon}$ is the variance of their intrinsic ellipticity distribution. We assume $n_{\mathrm{g}}=30$ and take into account that only such sources from the distribution (9) contribute to the signal whose redshift is larger than that of the lensing halo.

Convolving the projected NFW density profile (cf. Bartelmann 1996) with the weight function $U(|\boldsymbol{\vartheta}|)$, the aperture mass of NFW haloes as a function of halo mass, $M_{\mathrm{ap}}(M, \theta)$ is easily computed. Assuming further a halo mass function $N_{\text {halo }}(M, z)$, we can calculate the number of haloes per unit mass and redshift whose aperture mass is sufficiently high for the signal-to-noise ratio $\mathcal{S}$ to exceed a given threshold $\mathcal{S}_{\min }$. We choose the mass function suggested by Sheth \& Tormen (1999), which is a variant of the Press-Schechter (1974) mass function which well reproduces the mass function found in numerical simulations. We take into account that our definition of mass differs slightly from Sheth \& Tormen's in that we use the mass enclosed by a sphere in which the mean density is 200 times the critical rather than the mean density.

In calculating the number density of haloes of mass $M$ at redshift $z$ which produce a significant weak-lensing signal, $N_{\text {lens }}(M, z)$, we have to take into account that a signal-to-noise threshold $\mathcal{S}_{\min }$ for the weak-lensing signal does not correspond to an equally sharp threshold in halo mass because of the scatter in the aperture mass which is caused by the shot noise from the discrete background galaxy positions and their intrinsic ellipticity distribution. A halo of mass $M$ has a certain probability $p\left(M_{\mathrm{ap}} \mid M\right)$ to produce an aperture mass $M_{\mathrm{ap}}$, which we model as Gaussian,

$p\left(M_{\mathrm{ap}} \mid M\right) \propto \exp \left\{-\frac{\left[M_{\mathrm{ap}}-\hat{M}_{\mathrm{ap}}(M)\right]^{2}}{2 \sigma_{\mathrm{M}}^{2}}\right\}$, where $\sigma_{\mathrm{M}}$ is given by (11) and $\hat{M}_{\mathrm{ap}}(M)$ is the theoretical expectation for the aperture mass of a halo with mass $M$. The probability for a halo of mass $M$ to have an aperture mass above the signal-to-noise threshold is then

$P\left(\mathcal{S}>\mathcal{S}_{\min } \mid M\right)=\frac{1}{2} \operatorname{erfc}\left[\frac{\mathcal{S}_{\min }-\hat{\mathcal{S}}(M)}{\sqrt{2}}\right]$,

where $\operatorname{erfc}(x)$ is the complementary error function and $\hat{\mathcal{S}}(M)=$ $\hat{M}_{\text {ap }}(M) \sigma_{\mathrm{M}}^{-1}$. Thus, the number density of significantly lensing haloes is

$N_{\text {lens }}(M, z)=P\left(\mathcal{S}>\mathcal{S}_{\min } \mid M\right) N_{\text {halo }}(M, z)$.

We illustrate $N_{\text {lens }}(M, z)$ in Fig. 7. Here and below, we set $\mathcal{S}_{\min }=5$.

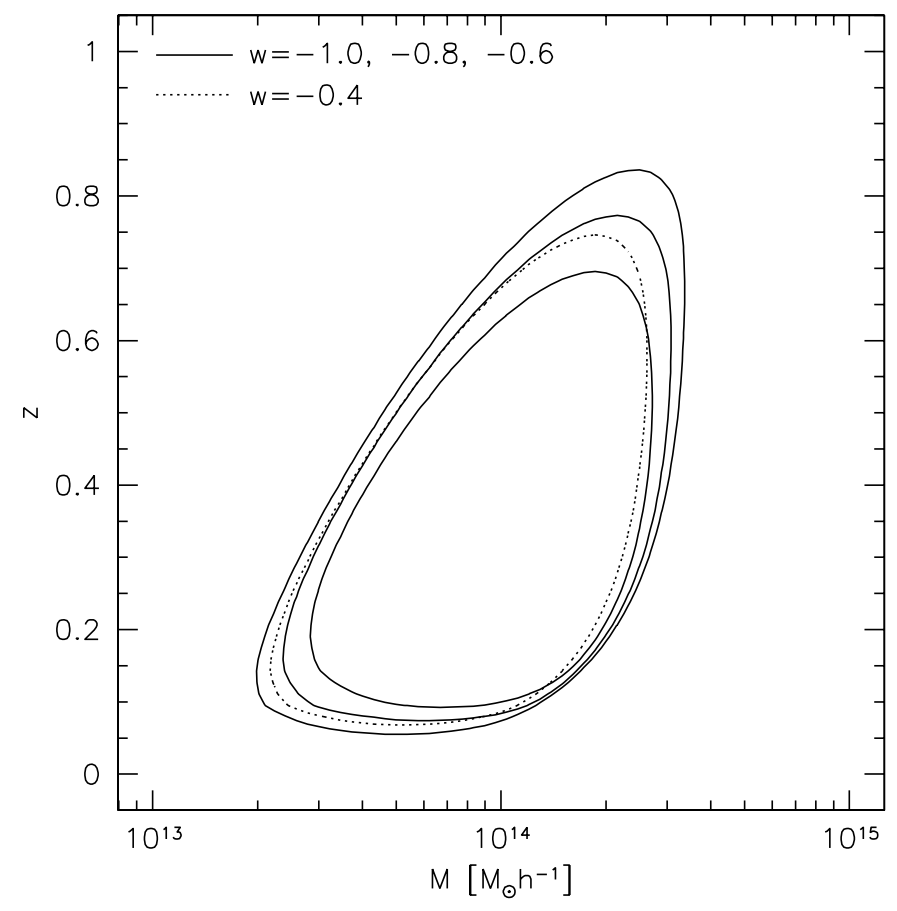

Fig. 7. Contours in the mass-redshift plane showing the number density of such haloes which are capable of producing a significant weaklensing signal with the aperture-mass technique. The contours are drawn at a single, arbitrarily chosen level corresponding to a halo density of $10^{-9} h M_{\odot}^{-1}$. The three solid contours, from inside out, show results for $w=-1.0,-0.8$ and -0.6 , respectively, while the dotted contour shows the result for $w=-0.4$. As $w$ increases above -1 , contours widen until $w \approx-0.6$, and shrink as $w$ increases further.

The figure shows two sets of contours, inner and outer, whose levels are $10^{-8.5}$ and $10^{-9.5} h M_{\odot}^{-1}$, respectively. Each set has four contours for different values of $w$, as indicated in the figure. All other cosmological parameters are kept fixed, i.e. $\Omega_{0}=0.3$ and $\Omega_{Q}=0.7$. The solid contour is for the $\Lambda \mathrm{CDM}$ model, for which $w=-1$. The dotted and shortdashed contours show that the region in the mass-redshift plane occupied by significantly lensing haloes widens as $w$ increases from -1 to -0.6 . If $w$ is increased further, this region shrinks considerably, as the long-dashed contour shows. This illustrates the competition between the two effects outline 


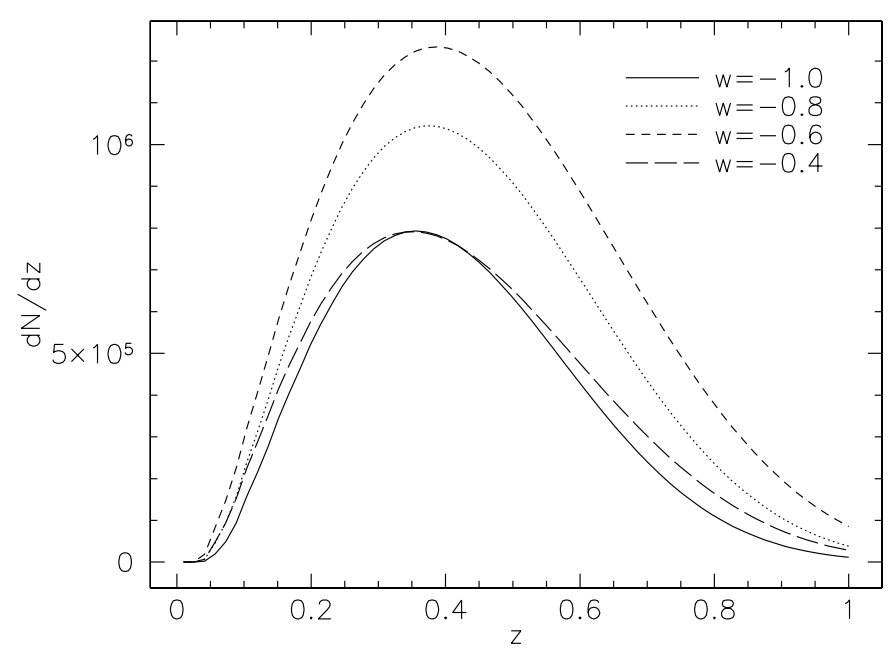

Fig. 8. The redshift distribution of weak-lensing haloes is shown for four different cosmological models, as indicated. As $w$ changes, the peak remains near $z \sim 0.4$, but the amplitude increases until $w \sim-0.6$ and drops for further increasing $w$.

above: haloes grow earlier and are thus more concentrated in dark-energy models with $w>-1$, but the integrated SachsWolfe effect reduces the power-spectrum normalisation required by the COBE-DMR data. The maximum extent of the contours in the mass-redshift plane is reached just before the strong decrease in $\sigma_{8}$ near $w \sim-0.6$ illustrated in Fig. 3 .

Figure 8 shows the redshift distribution of weak-lensing haloes for the same four dark energy models used for Fig. 7. The curves in Fig. 8 are thus integrals over mass of the distributions in Fig. 7,

$$
\frac{\mathrm{d} N_{\text {lens }}(z)}{\mathrm{d} z}=\int_{0}^{\infty} \mathrm{d} M N_{\text {lens }}(M, z) \text {. }
$$

As $w$ increases from $w=-1$ to $w=-0.6$, the amplitude of the redshift distribution rises by $\sim 50 \%$, and the redshift distribution extends towards higher redshift. At $w=-0.4$, the amplitude drops to the level of the $\Lambda$ CDM model $(w=-1)$, but the distribution is somewhat wider. This reflects the fact that the number of haloes is reduced compared to the models with somewhat lower $w$ because the power-spectrum normalisation is lower, but the haloes are more concentrated compared to the $\Lambda \mathrm{CDM}$ model because of their earlier formation, allowing them to be significant lenses at higher redshifts.

Finally, we show in Fig. 9 the total expected number of weak-lensing haloes per square degree as a function of $w$. The peak is reached with $\sim 17$ haloes per square degree at $w=-0.6$, which is almost a factor of two higher than for $\Lambda$ CDM models. Note also that the increase is roughly linear with $w$ up to the maximum. For larger values of $w$, the halo number drops steeply. However, as already stressed, these cases are not interesting for cosmology since they do not produce cosmic acceleration as required by observations.

\section{Summary and conclusions}

We investigated the expected properties of dark-matter haloes in dark energy cosmologies. For our purposes, the essential

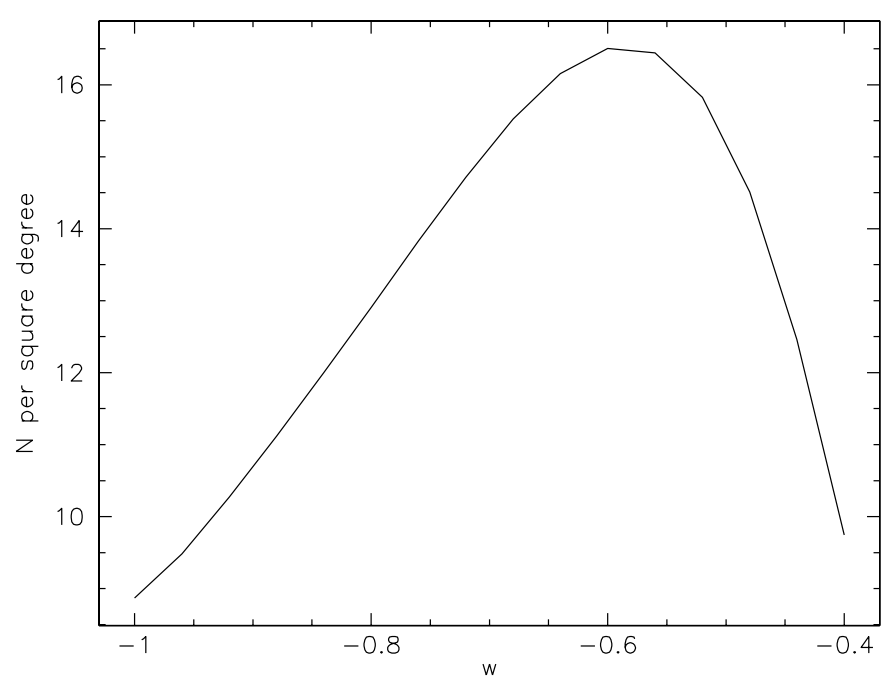

Fig. 9. Total number of significant weak-lensing haloes per square degree as a function of $w$. The curve reaches a peak near $w \sim-0.6$, where the halo number is approximately twice that for a $\Lambda \mathrm{CDM}$ model. For $w \gtrsim-0.6$, the halo number drops steeply.

features of such models are captured describing the dark energy as a density component with negative pressure $p_{\mathrm{Q}}=w \rho_{\mathrm{Q}}$, where $w \geq-1$ is a constant. The dark energy density $\rho_{\mathrm{Q}}$ is determined by its present value in units of the critical density, $\Omega_{\mathrm{Q}}$. In agreement with results from observations of the CMB, we focus on models which are spatially flat, $\Omega_{0}+\Omega_{\mathrm{Q}}=1$, have a matter density parameter $\Omega_{0}=0.3$, and a Hubble constant of $h=0.7$.

The modified background dynamics in dark-energy models has two immediate consequences. First, the growth factor is changed, which determines how structures grow against the expanding background. In models with fixed parameters $\Omega_{0}$ and $\Omega_{\mathrm{Q}}$, structures form earlier if $w$ is larger. Second, under equal circumstances, the cosmic volume shrinks as $w$ increases. Dark energy models thus "interpolate" between lowdensity, spatially flat models with cosmological constant and low-density, open models.

For our purposes, we can neglect the clustering of the quintessence field and assume that the dark-matter power spectrum is given by the common CDM spectrum. We take the shape parameter to be given by $\Gamma=\Omega_{0} h$ and normalise the spectrum such that the COBE-DMR measurements of CMB fluctuations on large angular scales are reproduced. This implies a third cosmological consequence. As $w$ increases, the gravitational potential of matter fluctuations evolves more rapidly along a given line of sight. Secondary anisotropies in the CMB caused by the integrated Sachs-Wolfe effect thus grow in amplitude. Keeping the total fluctuation amplitude fixed to the COBE-DMR data thus requires the amplitude of the primordial fluctuations to decrease. Expressing the powerspectrum normalisation by $\sigma_{8}$, this implies that $\sigma_{8}$ must decrease as $w$ increases. The decrease is gentle for $-1 \leq w \lesssim-0.6$ and steepens as $w$ increases further.

These findings have two counter-acting effects on the evolution of dark-matter haloes. First, haloes forming earlier are more concentrated because their core density reflects the density of the background universe at their formation time. 
Since structures form earlier in dark energy models as $w$ is increased, haloes are expected to become more concentrated as $w$ grows. Second, the decrease of $\sigma_{8}$ with increasing $w$ has the opposite effect on the halo formation time and indirectly on halo concentration. However, cosmologically interesting darkenergy equations of state must yield cosmic acceleration today and require $-1 \leq w \leq-0.6$. Within that range, the first effect is dominant, and the overall behaviour is monotonic.

Ideally, extensive, high-resolution numerical simulations would be necessary for quantifying the net result of these two effects. However, simple algorithms for calculating halo concentrations have already been derived from existing numerical simulations. We used them for our work, assuming that they are also valid with the modification of Friedmann's equation caused by the introduction of dark energy instead of a cosmological constant.

We used three different recipes for computing halo concentrations. Albeit differing in detail, they agree in concept. Haloes are assigned a formation epoch, essentially requiring that a certain fraction of the final halo mass has already collapsed into sufficiently massive progenitors. The characteristic density of the haloes is then taken to be proportional to the mean background density of the universe at the halo formation epoch. We showed that all three recipes lead to the result that haloes are expected to be increasingly more concentrated as $w$ grows in quintessence models, showing that the effect of their earlier growth is stronger than the effect of decreasing $\sigma_{8}$. This holds for $w \lesssim-0.6$ and reverses for larger $w$ because the integrated Sachs-Wolfe effect then requires a steep decrease in $\sigma_{8}$. The particular recipe for computing halo concentrations described by Bullock et al. implies that haloes should be $\sim 50 \%$ more concentrated for $w=-0.6$ than for $w=-1$, where the increase is roughly linear with $w$.

Finally, we described that halo searches using weak-lensing techniques are sensitive to halo concentrations. Using the Sheth-Tormen modification of the Press-Schechter mass function for quantifying the halo population in mass and redshift, and the aperture mass technique for quantifying the weaklensing effects of haloes, we showed that the expected number density on the sky of haloes causing 5- $\sigma$ weak-lensing detections approximately doubles as $w$ increases from -1 to -0.6 , where the increase is linear with $w$. Our results indicate that halo concentrations may be a sensitive probe for the darkenergy equation of state, and that gravitational lensing may provide the observational tools for applying that probe.

Note, however, that we did not allow variations in some cosmological parameters which may also change the number of weak-lensing haloes. In particular, an effect may arise from varying the index $n$ of the dark-matter power spectrum because it directly affects the determination of $\sigma_{8}$. On the other hand, our approach here is to characterise the main effects of dark energy on halo formation and to propose weak lensing studies as a tool for constraining the dark energy itself, assuming the main cosmological parameters will be measured by independent observations like those of the CMB.

Thus, weak lensing turns out to be a powerful tool not only for mapping the distribution of matter in the Universe, but also for probing fundamental dark-energy properties. Currently, weak lensing observations do not allow detailed reconstructions of halo density profiles (Mellier 2001; Clowe et al. 2000; Mellier \& van Waerbeke 2001), mainly because of the resolution limit due to the finite number density of background galaxies.

On the other hand, interesting new perspectives have been opened by several recent wide-field cosmic-shear studies (Bacon et al. 2000; Bacon et al. 2002; Wittman et al. 2000; van Waerbeke et al. 2000; Kaiser et al. 2000). Future weak lensing surveys will cover even larger fields and, thanks to the improved control of systematic errors, they will allow tighter constraints on the cosmological parameters. Besides the wide field telescopes which are currently in their project study phases, we specifically mention the "dark matter telescope" LSST (http://dmtelescop.org, proposed to scan a 7 square-degree sky field), the VISTA survey (http://www.vista.ac.uk), and the SNAP satellite (http://snap.lbl.gov), whose weak lensing survey will cover an area of 300 square degrees, resulting in a very wide field survey with excellent image quality and depth.

Acknowledgements. We thank Uroš Seljak and Simon White for valuable discussions.

\section{References}

Armendariz-Picon, C., Mukhanov, V., \& Steinhardt, P. J. 2001, Phys. Rev. D, 63, 3510

Baccigalupi, C., Matarrese, S., \& Perrotta, F. 2000, Phys. Rev. D, 62, 123510

Baccigalupi, C., Balbi, A., Matarrese, S., Perrotta, F., \& Vittorio, N. 2002, Phys. Rev. D, 65, 3520

Bacon, D., Refregier, A., \& Ellis, R. 2000, MNRAS, 318, 625

Bacon, D., Massey, R., Refregier, A., \& Ellis, R. 2002, preprint [astro-ph/0203134]

Bartelmann, M. 1996, A\&A, 313, 697

Bartelmann, M., \& Schneider, P. 2001, Phys. Rep., 340, 291

Bardeen, J. M., Bond, J. R., Kaiser, N., \& Szalay, A. S. 1986, ApJ, 304, 15

Bennett, C. L., Banday, A. L., Gorski, K. M., et al. 1996, ApJ, 464, L1

Bullock, J. S., Kolatt, T. S., Sigad, Y., et al. 2001, MNRAS, 321, 559

Bunn, E. F., \& White, M. 1997, ApJ, 480, 6

Clowe, D., Luppino, G., Kaiser, N., \& Gioia, I. M. 2000, ApJ, 539, 540

Chiba, T. 2001, Phys. Rev. D, 64, 103503

Cohen, J. G., Hogg, D. W., Blandford, R. D., et al. 2000, ApJ, 538, 29

Corasaniti, P., \& Copeland, E. J. 2002, Phys. Rev. D, 65, 043004

De Bernardis, P., Ade, P. A. R., Bock, J. J., et al. 2002, ApJ, 564, 559

Dodelson, S., Kaplinghat, M., \& Stewart, E. 2000, Phys. Rev. Lett., 85,5276

Doran, M., Lilley, M., \& Wetterich, C. 2002, Phys. Lett. B, 528, 175

Eke, V. R., Navarro, J. F., \& Steinmetz, M. 2001, ApJ, 554, 114

Futamase, T., \& Yoshida, S. 2001, Progr. Theor. Phys., 105, 887

Halverson, N. W., Leitch, E. M., Pryke, C., et al. 2002, ApJ, 568, 38

Heath, D. 1977, MNRAS, 179, 351

Hu, W. 1998, ApJ, 506, 485

Huterer, D. 2002, Phys. Rev. D, 65, 063001

Kaiser, N., Wilson, G., \& Luppino, G. A. 2000, ApJ, in press, preprint [astro-ph/0003338]

Kruse, G., \& Schneider, P. 1999, MNRAS, 302, 821

Lee, A. T., Ade, P., Balbi, A., et al. 2001, ApJ, 561, L1 
Liddle, A. R., \& Scherrer, R. J. 1999, Phys. Rev. D, 59, 3509

Łokas, E. L., \& Hoffmann, Y. 2002, MNRAS, submitted, preprint [astro-ph/0108283]

Ma, C. P., Caldwell, R. R., Bode, P., \& Wang, G. L. 1999, ApJ, 521, L1

Maoli, R., van Waerbeke, L., Mellier, Y., et al. 2001, A\&A, 368, 766

Mellier, Y. 1999a, ARA\&A, 37, 127

Mellier, Y. 1999b, Proc. of the NATO Advanced Study Institute on Theoretical and Observational Cosmology, ed. M. Lachieze-Rey (Kluwer Academic Press) [astro-ph/99001116]

Mellier, Y. 2001, Proc. of the ISSI workshop Matter in the Universe, vol. 15, ed. Ph. Jetzer, K. Pretzl, \& R. von Steiger (Kluwer Academic Press)

Mellier, Y., \& van Waerbeke, L. 2001, Where's the matter? Tracing Dark and Bright Matter with the New Generation of Large-Scale Surveys, Proc. Conf. June 2001, ed. Treyer \& Tresse (Frontier Group)

Navarro, J. F., Frenk, C. S., \& White, S. D. M. 1995, MNRAS, 275, 720

Navarro, J. F., Frenk, C. S., \& White, S. D. M. 1997, ApJ, 490, 493

Peacock, J. A. 1999, Cosmological Physics (Cambridge: University Press)
Peacock, J. A., \& Dodds, S. J. 1996, MNRAS, 280, L19

Percival, W. J., Baugh, C. M., Bland-Hawthorn, J., et al. 2001, MNRAS, 327, 1297

Perlmutter, S., Aldering, G., Goldhaber, G., et al. 1999, ApJ, 517, 565

Press, W. H., \& Schechter, P. 1974, ApJ, 187, 425

Ratra, B., \& Peebles, P. J. E. 1988, Phys. Rev. D, 37, 3406

Riess, A. G., Filippenko, A. V., Challis, P., et al. 1998, AJ, 116, 1009

Schneider, P. 1996, MNRAS, 283, 837

Seljak, U., \& Zaldarriaga, M. 1996, ApJ, 469, 437

Sheth, R. K., \& Tormen, G. 1999, MNRAS, 308, 119

Smail, I., Hogg, D. W., Yan, L., \& Cohen, J. G. 1995, ApJ, 449, L105

Steinhardt, P. J., Wang, L., \& Zlatev, I. 1999, Phys. Rev. D, 59, 3504

Tocchini-Valentini, D., \& Amendola, L. 2002, Phys. Rev. D, 65, 3508

Tyson, J. A., Valdes, F., Jarvis, J. F., Mills, A. P. Jr. 1984, ApJ, 281, L59

van Waerbeke, L., Mellier, Y., Erben, T., et al. 2000, A\&A, 358, 30

van Waerbeke, L., Mellier, Y., Radovich, M., et al. 2001, A\&A, 374, 757

Wang, L., \& Steinhardt, P. J. 1998, ApJ, 508, 483

Wetterich, C. 1988, Nucl. Phys. B, 302, 645

Wittmann, D. M., Tyson, J. A., Kirkman, D., Dell'Antonio, I., \& Bernstein, G. 2000, Nature, 405, 143 